\title{
PREPARATION AND CHARACTERIZATIONS OF NEW BIOMATERIALS BY ANTHOCYANINS ADSORPTION ON HYDROXYAPATITE-BASED MATERIALS
}

\author{
RÉKA BARABÁS ${ }^{a}$, NORBERT MUNTEANa ${ }^{a}$, GABRIELLA SZABÓa, \\ KRISZTINA MAURER ${ }^{\mathrm{a}}$, LILIANA BIZO ${ }^{\mathrm{b} *}$
}

\begin{abstract}
Anthocyanins (ACNs) are water-soluble plant pigments belonging to the group of flavonoids, with antioxidant, anti-inflammatory and antibacterial activity. The antioxidant activities of extracts from berries and hibiscus were tested with the Briggs-Rauscher method. Adsorption of anthocyanins on biomaterials is a method for their stabilization. Hydroxyapatite (HAP) doped with inorganic silica has an increased specific surface, porosity and chemical reactivity. Anthocyanins from extracts of different berries were adsorbed on hydroxyapatite-based materials. The adsorption and desorption capacity of hydroxyapatite-based materials were measured and the adsorption capacity of different anthocyanin extracts were compared. It was concluded that the highest adsorption capacity and efficiency were obtained in the case of blueberry. Based on the results of the desorption experiments, it has been proven that HAP-Si-blueberry is more adequate to obtain a retard effect, despite the fact that the antioxidant activities of blueberry and blackcurrant are similar.
\end{abstract}

Keywords: anthocyanin, antioxidant activity, hydroxyapatite-based materials, berries extract, hibiscus extract.

\section{INTRODUCTION}

Silica doped hydroxyapatite was mainly used for biomedical purposes. Besides biocompatibility, hydroxyapatite shows a very high reactivity with metal ions, which can lead to the use of this material in new application fields,

a Babeş-Bolyai University, Faculty of Chemstry and Chemical Engineering, Department of Chemistry and Chemical Engineering of the Hungarian Line, 11 Arany Janos str., RO400028, Cluj-Napoca, Romania

b Babeş-Bolyai University, Faculty of Chemstry and Chemical Engineering, Department of Chemical Engineering, 11 Arany Janos str., RO-400028, Cluj-Napoca, Romania

*Corresponding author Ibizo@chem.ubbcluj.ro 
for example using water purification agents for heavy metal immobilization. Based on its ion exchange property (regarding calcium and other metal ions), HAP is widely used as a metal retainer material [1].

Due to the introduction of silica, the specific surface, porosity and in this way the chemical reactivity of the apatite material is enlarged; doping the hydroxyapatite with silica can be a better material for metal removal [2-4]. HAP doped with inorganic silica has an increased specific surface, porosity and chemical reactivity. Heat-treated materials have a very low specific surface area, which explains the weak metal sorption properties of these materials. Materials containing silica have better sorption efficiency than unmodified hydroxyapatite and $10 \% \mathrm{wt}$ silica-containing hydroxyapatite with low particle size has the highest copper sorption efficiency. Increase of the initial copper concentration decreases saturation time and increase of temperature increases copper sorption. Silica-containing hydroxyapatite shows very good copper sorption capacity, which can lead to the enlargement of the application fields of apatites [5].

\section{Definition of antioxidant effect (AOX)}

Anthocyanins are water-soluble phytochemicals with a typical red to blue color. Anthocyanins belong to the group of flavonoids, they can be found in tea, honey, wine, fruits, vegetables, nuts, olive oil, cocoa and cereals. Anthocyanin extracts from plants are promising as a treatment for hypertension and hyperlipidemia [6]. Many authors have also reported about the antioxidant activity [7], anti-inflammatory activity [8, 9], cardioprotective activity [10], hepatoprotective activity [11] and antibacterial activity [12] of anthocyanins.

Antioxidant activity denotes the ability of a bioactive compound to maintain cell structure and function by effectively clearing free radicals, inhibiting lipid peroxidation reactions and preventing other oxidative damage [13]. It is also a foundation of many other biological functions, such as anti-cancers, antiinflammation and anti-aging $[14,15]$. On the other hand, the prevention of many chronic diseases, such as cancer, diabetes and cardiovascular disease, has been suggested to be associated with the antioxidant activity $[16,17]$.

\section{Antioxidant activity measurements (AOX)}

Therefore, a deep study of natural antioxidants, such as those from fruits and vegetables, is of great importance to human health. There are different antioxidant testing methods, for example ORAC (oxygen radical absorbance capacity), TRAP (total radical trapping antioxidant parameter), TEAC (Trolox equivalent antioxidant capacity), FRAP (ferric ion reducing antioxidant parameter), reaction with DPPH (2, 2-diphenyl-1-picrylhydrazyl), TOSC (total oxidant scavenging capacity), etc., which provide different ranking orders for antioxidant capacity due to different experimental conditions [18-20]. 
In this study the Briggs-Rauscher method (BR) was used, which is based on the inhibition of the oscillations due to the antioxidants reaction with the free radical intermediates. This method [21] was chosen because it works at $\mathrm{pH} 2$ and partially mimics the physiological conditions similar to those of the fluids in the human stomach, and it is applicable for the investigation of hydrophilic as well as lipophilic compounds [22, 23]. The Briggs-Rauscher method was described by Cervellati et al. (2001) and it has been widely used since then [24-27]. Briefly, when antioxidants are added to an active BR mixture, there is an immediate quenching of the oscillations and the time elapsed between the cessation and the return of the oscillating regime, the so called inhibition time. The dependence of the inhibition time on the antioxidant concentration is suitable for determination of the antioxidant capacity. The slope of the curve representing inhibition time vs. antioxidant concentration (inhibition time versus concentration) can be used to calculate the relative antioxidant activity. The relative activity with respect to the slopes (RAS) of a compound is the ratio between the slope of the straight line of the respective compound and that of the chosen standard: RAS = slope (sample)/ slope (standard), so that a high RAS number means a better antioxidant effectiveness. The efficiency of the corresponding antioxidant is expressed as the length of time in minutes before oscillations restart. A prolonged inhibition time ( tinhib. $_{\text {. }}$ means a better antioxidant activity.

Adsorption of anthocyanins on biomaterials is a method for their stabilization. Hydroxyapatite (HAP) is an important biomaterial, although it does not possess all the necessary properties to be the perfect biomaterial (mechanical strength, chemical reactivity, etc.). Silica is also a component of the bone; therefore, several methods for the synthesis of silicon-substituted hydroxyapatites have been described.

The aim of this work was the extraction of anthocyanins with AOX effect and their adsorption and desorption on hydroxyapatite (HAP) and silicasubstituted hydroxyapatite (HAP-Si). Anthocyanins stabilized by adsorption on HAP-based materials should be applied in stomatology as anti-inflammatory treatments to avoid the inflammation of the cardiac system. Consequently, our research was focused on:

1. Extracting antioxidants from hibiscus and different berries; separation of anthocyanins from hibiscus extract and comparison of their antioxidant activity (the antioxidant activity was measured using the BR analytical method based on the inhibition of the Briggs-Rauscher oscillating reaction),

2. Testing the adsorption capacity of anthocyanin extracts on HAP-Si, 
3. Testing the antioxidant activity of extracts from hibiscus and anthocyanins from hibiscus extract,

4. Testing the antioxidant activity of extracts from berries (blueberry, hawthorn, blackcurrant, blackthorn).

\section{RESULTS AND DISCUSSION}

\section{Antioxidant activity evaluation}

Perturbation of the oscillatory BR system with a diluted extract causes the immediate cessation of the oscillations; the time elapsed between cessation and returns of oscillations (the so called inhibition time) are illustrated in Figure 1.

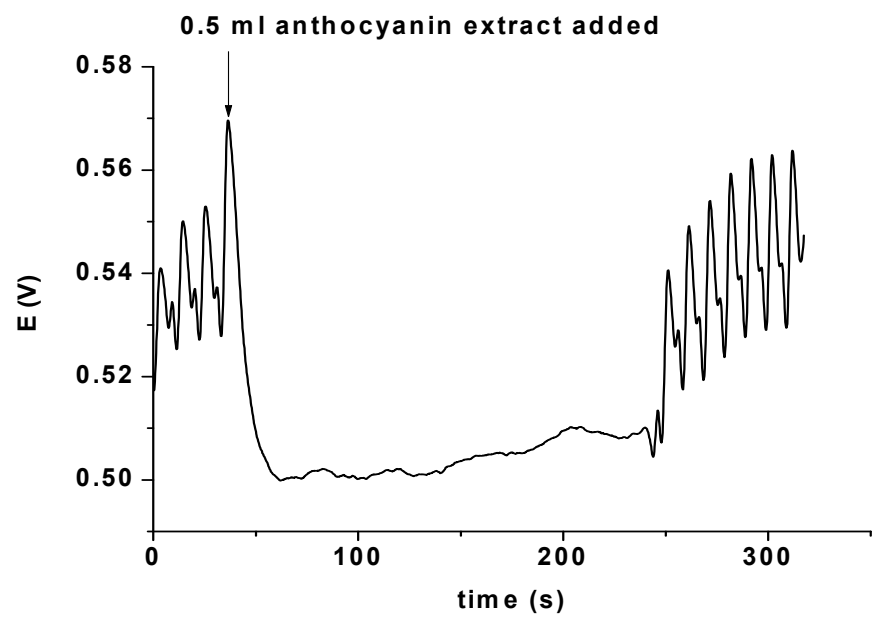

Figure 1. The effect of $A E H$ extract on $B R$ oscillating reaction.

\section{Analysis of AOX activities of AEH and HE}

Preliminary experiments regarding the AOX activity of anthocyanins were made in the case of AEH (anthocyanin from hibiscus extract) and HE (hibiscus extract). Stock solutions of $\mathrm{HE}$ and $\mathrm{AEH}$ were prepared by dissolving $0.001 \mathrm{~g}$ of extract in $10 \mathrm{ml}$ of distilled water. The antioxidant activity was determined for several dilutions and the calibration curves were drawn for each of them. Variation of the inhibition time depending on the antioxidant concentration was found to be linear as can be seen in the figure below (Figure 2). 
The relative antioxidant effect can be calculated from the slope of the calibration curves (see next paragraph). The relative activity with regard to slopes (RAS) is the ratio between the slope of the straight line of the sample and that of the standard; i.e. RAS = slope (sample)/slope (standard). As listed in Table 1, the chosen standard was the pure anthocyanin with a slope of 656.8 , the slope of the extract calibration curve was 1371.1, the RAS of the extract was 2.1, thus the extract had more than double antioxidant capacity than the pure separated anthocyanin. This superior behavior is probably caused by the synergistic effect of other compounds present in the extract.

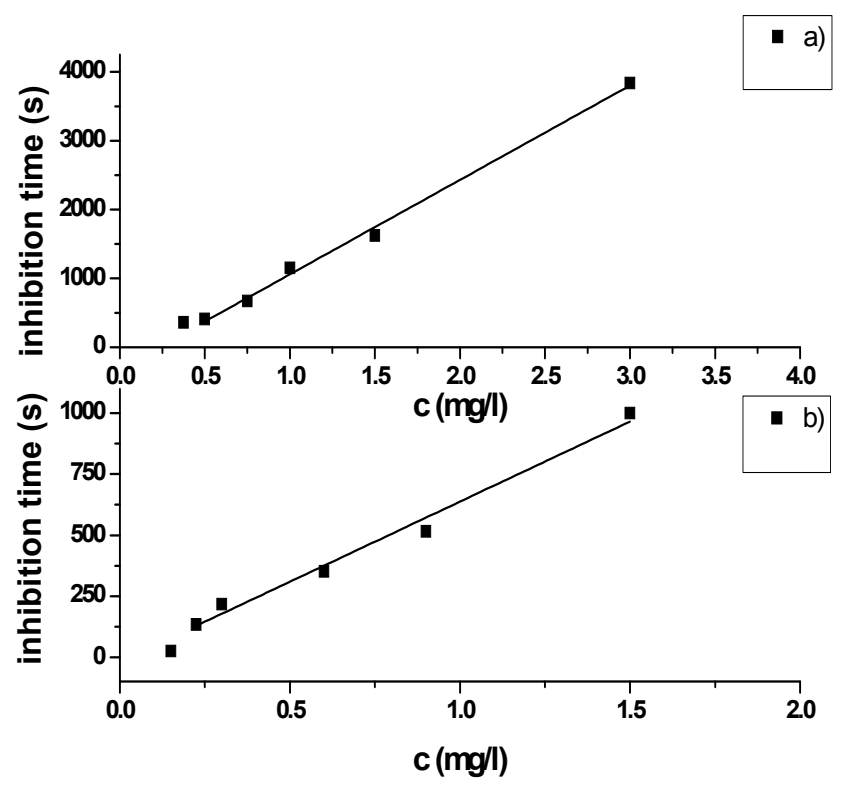

Figure 2. Concentration dependence of the inhibition time for: a) hibiscus extract; b) anthocyanin from hibiscus extract.

Table 1. Relative activity calculated for hibiscus extract (HE) and for anthocyanin from hibiscus extract (AEH).

\begin{tabular}{|c|c|c|}
\hline Hibiscus & Slope of calibration curves & RAS \\
\hline $\mathrm{HE}$ & 1371.1 & 2.1 \\
\hline $\mathrm{AEH}$ & 656.8 & 1 \\
\hline
\end{tabular}


Testing the antioxidant activity of different types of berry extracts

The antioxidant activities of different AOX extracts were determined for several dilutions and the calibration curves were drawn for each of them. Variation of the inhibition time depending on the antioxidant concentration was found to be linear as can be seen in Figure 3 .

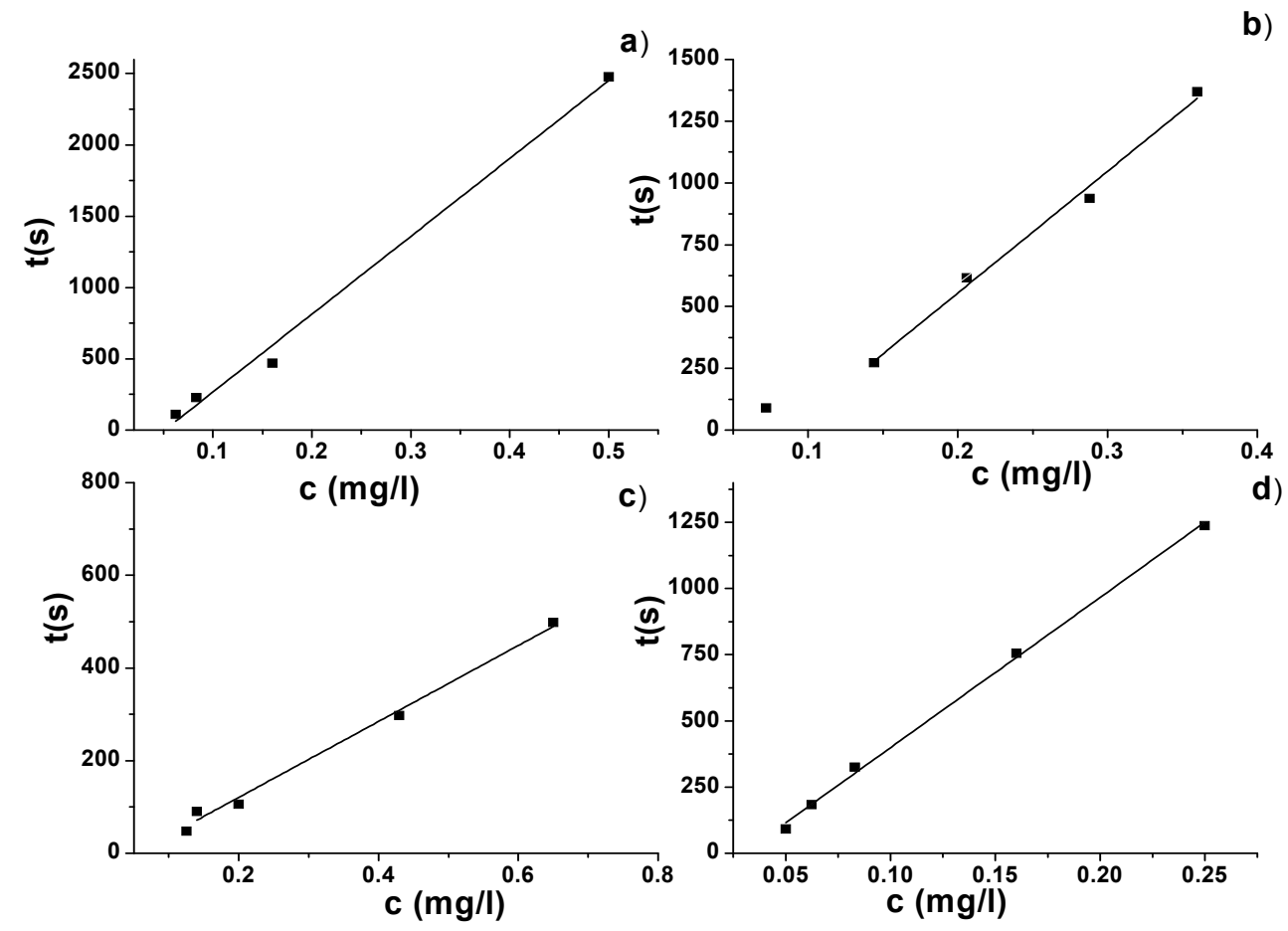

Figure 3. Concentration dependence of the inhibition time for: a) blueberry extract; b) hawthorn extract; c) blackthorn extract; d) blackcurrant extract.

Relative antioxidant activity was calculated using the slopes of the calibration curves of the berry extracts. The chosen standard was blackcurrant. The results are summarized in Table 2. 
Table 2. Relative antioxidant activity calculated for different types of berry extracts.

\begin{tabular}{|c|c|c|}
\hline Berry extracts & $\begin{array}{c}\text { Slope of calibration } \\
\text { curves }\end{array}$ & RAS \\
\hline Blackcurrant & 5673.9 & 8.6 \\
\hline Blueberry & 5456.3 & 8.3 \\
\hline Hawthorn & 4475.7 & 6.8 \\
\hline Blackthorn & 856.98 & 1.3 \\
\hline
\end{tabular}

Based on the RAS values calculated, the following ranking of antioxidant activity could be concluded:

\section{Blackcurrant $\approx$ Blueberry > Hawthorn $\gg$ Blackthorn}

There is no significant difference between blackcurrant and blueberry.

\section{Adsorption results}

Preliminary experiments carried out with hibiscus and regarded to the extraction of anthocyanins and measurements of AOX activity showed that the adsorption capacity of the hibiscus extract (anthocyanins and other compounds) was higher than that of the pure anthocyanins (without other accompanying components), see Figure 4.
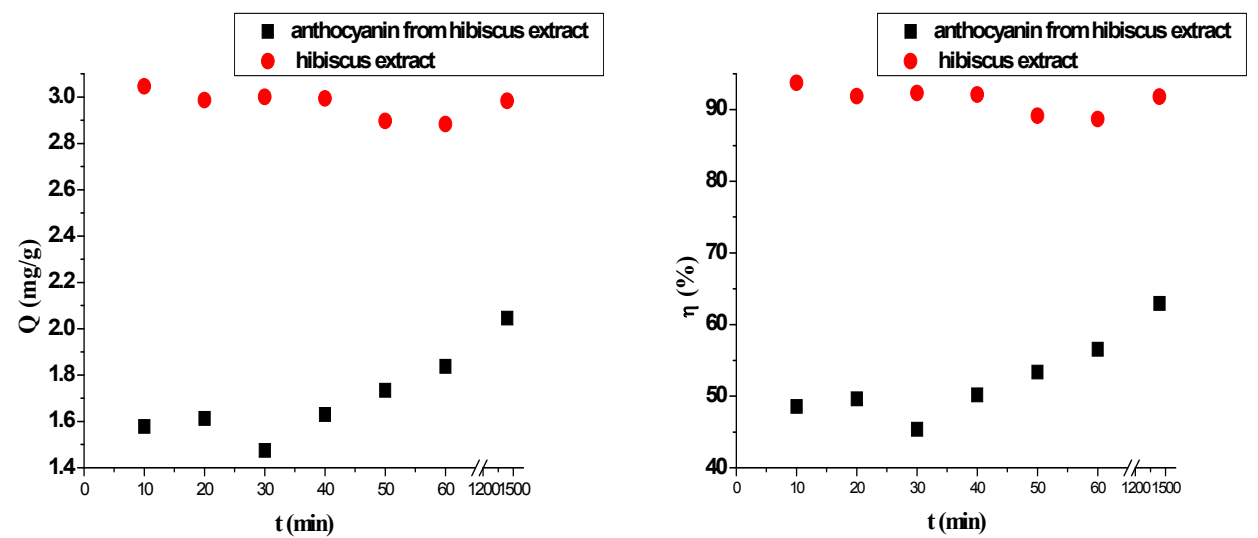

Figure 4. The adsorption capacity $q_{t}(\mathrm{mg} / \mathrm{g})$ and the adsorption efficiency $\eta(\%)$ of HAP-Si.

Based on these results, the adsorption experiments were carried out with berry extracts from: blueberry, hawthorn, blackthorn, and blackcurrant. 
The following table (Table 3) contains the adsorption capacity and adsorption efficiency of HAP-Si measured for extracts of different types of berries, obtained after 24 hours - time necessary to achieve the equilibrium.

Table 3. Adsorption capacity $\mathrm{q}_{\mathrm{t}}(\mathrm{mg} / \mathrm{g})$ and adsorption efficiency $\eta(\%)$ of HAP-Si measured for extracts of different types of berries.

\begin{tabular}{|c|c|c|}
\hline $\begin{array}{c}\text { Anthocyanin } \\
\text { mixture from: }\end{array}$ & $\begin{array}{c}\text { Adsorption capacity } \\
\boldsymbol{q}_{\boldsymbol{t}}(\mathrm{mg} / \mathrm{g}) \text { of HAP-Si }\end{array}$ & $\begin{array}{c}\text { Adsorption efficiency } \boldsymbol{\eta} \\
(\%) \text { of HAP-Si }\end{array}$ \\
\hline Blueberry & 50.29 & 41.91 \\
\hline Hawthorn & 45.06 & 37.55 \\
\hline Blackcurrant & 32.52 & 27.10 \\
\hline Blackthorn & 13.09 & 10.91 \\
\hline
\end{tabular}

It can be concluded (see Table 3), that the highest adsorption capacity and efficiency were obtained in the case of blueberry. Differences between adsorption capacities and efficiencies can be explained with the different types of anthocyanins found in the berries.

\section{Desorption experiments}

\section{Preparation of HAP-Si-AOX tablets}

For desorption experiments, HAP-Si powder with anthocyanins adsorbed from berries was used. Samples were prepared using a Specac hydraulic presser to obtain tablets on which surfaces anthocyanins were adsorbed. After the tablets were made, the adsorbed anthocyanin was calculated from the adsorption capacity defined in the previous experiments (see Table 4).

Table 4. Calculated adsorbed anthocyanin ( $\mathrm{mg}$ and \%) from HAP-Si-AOX tablets.

\begin{tabular}{|c|c|c|c|}
\hline Berries & Tablet weight $(\mathrm{g})$ & $\begin{array}{c}\text { Adsorbed } \\
\text { anthocyanin }(\mathrm{mg})\end{array}$ & $\begin{array}{c}\text { Adsorbed } \\
\text { anthocyanin \% }\end{array}$ \\
\hline Blueberry & 0.1345 & 6.76 & 5.0 \\
\hline Hawthorn & 0.1296 & 5.84 & 4.5 \\
\hline Blackcurrant & 0.1413 & 4.59 & 3.2 \\
\hline Blackthorn & 0.3383 & 4.43 & 1.3 \\
\hline
\end{tabular}




\section{Desorption of anthocyanins from HAP-Si-AOX tablets}

Desorption experiments were carried out as follows: tablets were immersed in simulated body fluid $[28,29]$ and the process was monitored by using a UV-VIS spectrophotometer. The desorbed amount of anthocyanin was calculated from the calibration curves of each extract. Desorption of the active compound from tablets is presented in Figure 5.

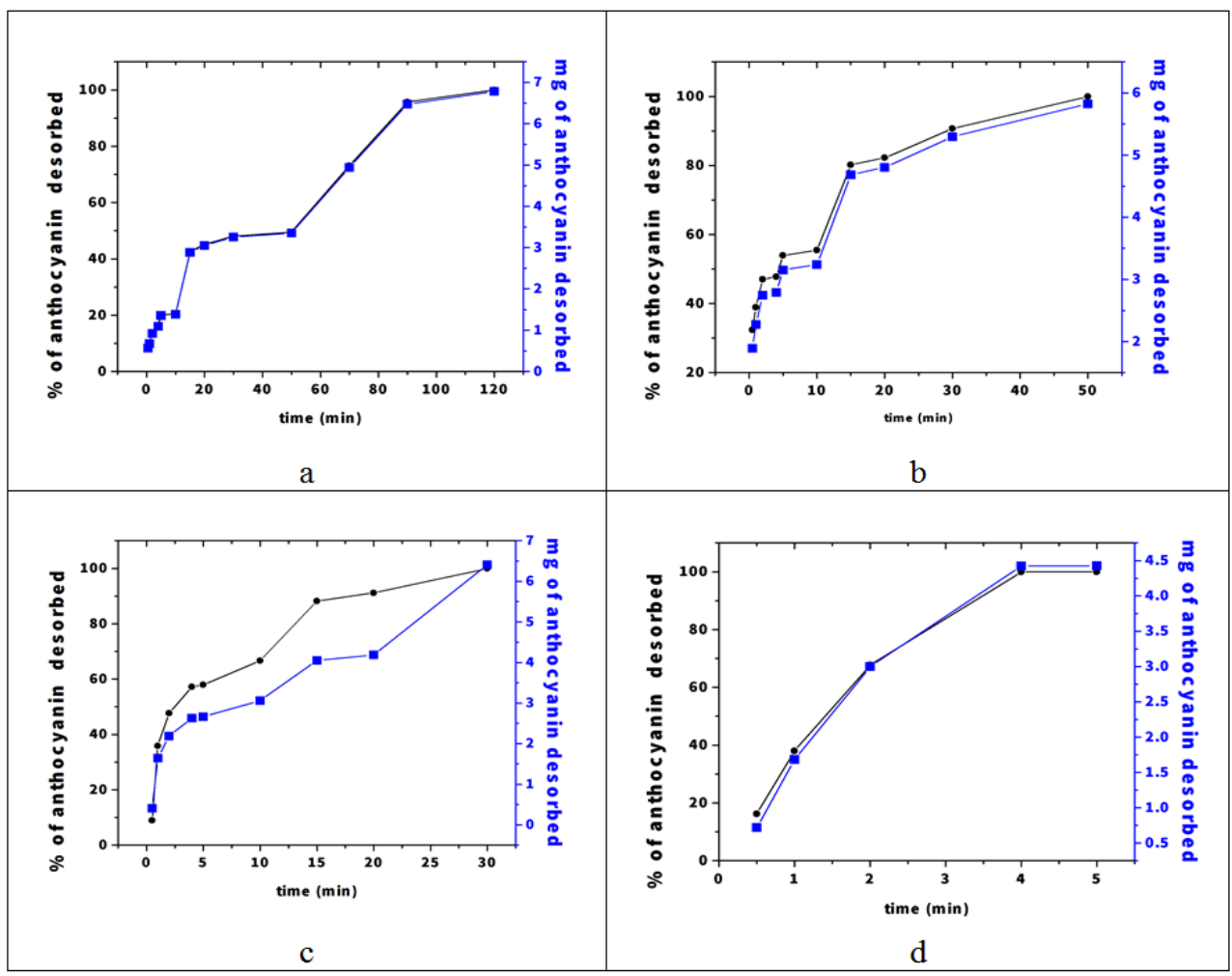

Figure 5. The amounts (blue line) and percentages (black line) of desorbed anthocyanins were plotted versus time in the case of: a) blueberry; b) hawthorn; c) blackcurrant; d) blackthorn.

Based on the desorption curves, the half-life values of the HAP-Sianthocyanin samples could be predicted, as summarized in Table 5. The results of the desorption experiments were well correlated with the adsorption experiments: the HAP-Si-blueberry sample had the highest adsorption capacity and the longest retention time of the adsorbed material at the same time. The order of adsorption capacity and the order of half-life were the same. 
Table 5. Predicted half-life values of the HAP-Si-anthocyanins.

\begin{tabular}{|c|c|}
\hline HAP-Si-anthocyanins & Predicted half-life (min) \\
\hline HAP-Si-blueberry & 40 \\
\hline HAP-Si-hawthorn & 15 \\
\hline HAP-Si-blackcurrant & 10 \\
\hline HAP-Si-blackthorn & 2 \\
\hline
\end{tabular}

On the other hand, the antioxidant activities of blueberry and blackcurrant were similar (see Table 2), but the half-life values of the samples were different: HAP-Si-blueberry was more adequate to obtain a retard effect.

\section{Transmission Electron Microscopy (TEM) measurements}

Transmission electron microscopy was performed on HAP-Si and HAP-Si-AOX (HAP-Si powder with adsorbed anthocyanins from berries) samples. The resulted images are presented in Figure 6. As the TEM pictures show, there is a difference between HAP-Si and HAP-Si-AOX; the latter presents gelified materials (berry extract) containing polysaccharides and anthocyanins.
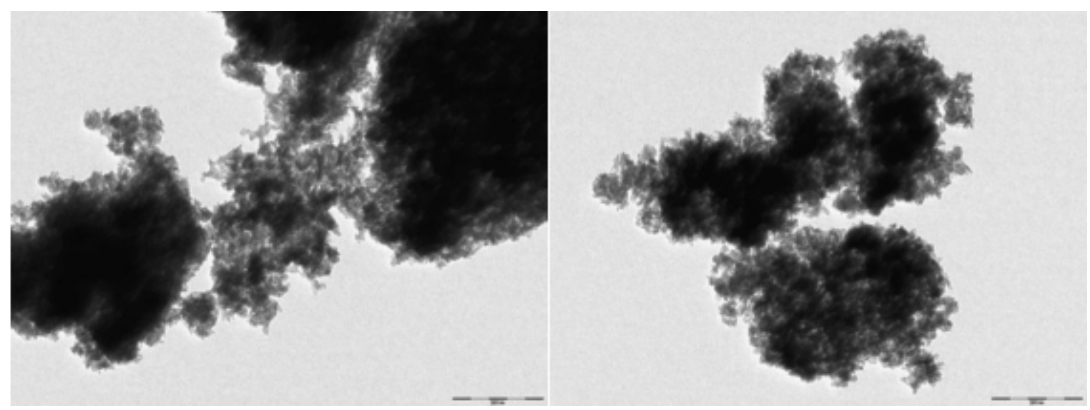

(a)

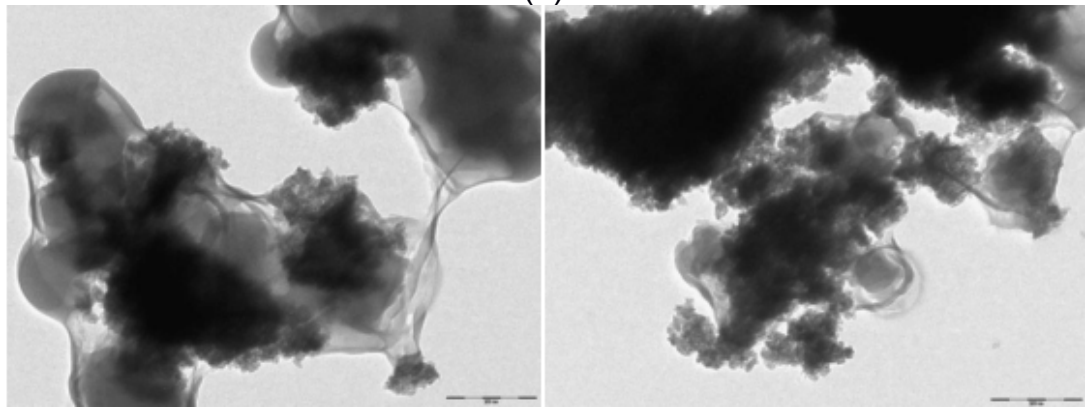

(b)

Figure 6. TEM images at 200nm resolution of (a) HAP-Si and (b) HAP-Si-AOX (HAP-Si powder with adsorbed anthocyanins from berries). 
PREPARATION AND CHARACTERIZATIONS OF NEW BIOMATERIALS BY ANTHOCYANINS ...

\section{CONCLUSIONS}

It can be concluded, that the adsorption capacity of hibiscus extract (anthocyanins and other compounds) was higher than that of the separated anthocyanins (without other accompanying components), due to the synergistic effect.

Based on the first results (with hibiscus), further experiments were carried out with various berries using berry extracts containing anthocyanins and other compounds (such as polysaccharides). These extracts were compared based on their antioxidant activity and the following ranking was made: Blackcurrant $\approx$ Blueberry > Hawthorn $>>$ Blackthorn.

The resulted extracts were adsorbed on HAP-Si, and the adsorption capacity and efficiency of each were evaluated. Based on the results it can be concluded, that the HAP-Si of the blueberry extract had the highest adsorption capacity and efficiency.

The desorption experiments in SBF showed that the HAP-Siblueberry composite had the highest half-life value. Due to these favorable properties, this composite can be used as supplement or dental filling/inlay with high antioxidant activity.

\section{EXPERIMENTAL SECTION}

\section{Extraction and separation of anthocyanins from different plants}

\section{Preparation of hibiscus extract (HE) by ultrasound-assisted extraction method}

The hibiscus (Hibiscus sabdariffa L.) petals used in this study is a bioproduct obtained from a Hungarian company. The powder of dried and mild hibiscus petals was mixed with different solvents in a conical flask. The conical flask was immersed in an ultrasonic bath at room temperature for 30 minutes. To avoid the oxidation of anthocyanins, nitrogen gas was led in the flask to ensure an oxygen-free environment. Vacuum filtration was performed, and the filtered material was extracted from the filter and treated with solvent in the ultrasonic bath for 30 minutes. The filtrate was transferred into a single-neck flask and the solvent removed by a rotary evaporator using low temperature $\left(40^{\circ} \mathrm{C}\right)$ and pressure to avoid the degeneration of the anthocyanin molecules. The filtrate was placed in the refrigerator to freeze overnight and then placed in the freeze drying apparatus to get rid of water. The hibiscus extract resulted from the above presented method will be denoted: HE. 


\section{Extraction of anthocyanin from hibiscus extract (AEH)}

Separation by solid phase extraction was used to extract pure anthocyanin from hibiscus extract. Oszmianski and Lee [30] developed this method and the steps of the extraction used in our study are summarized in Figure 7.

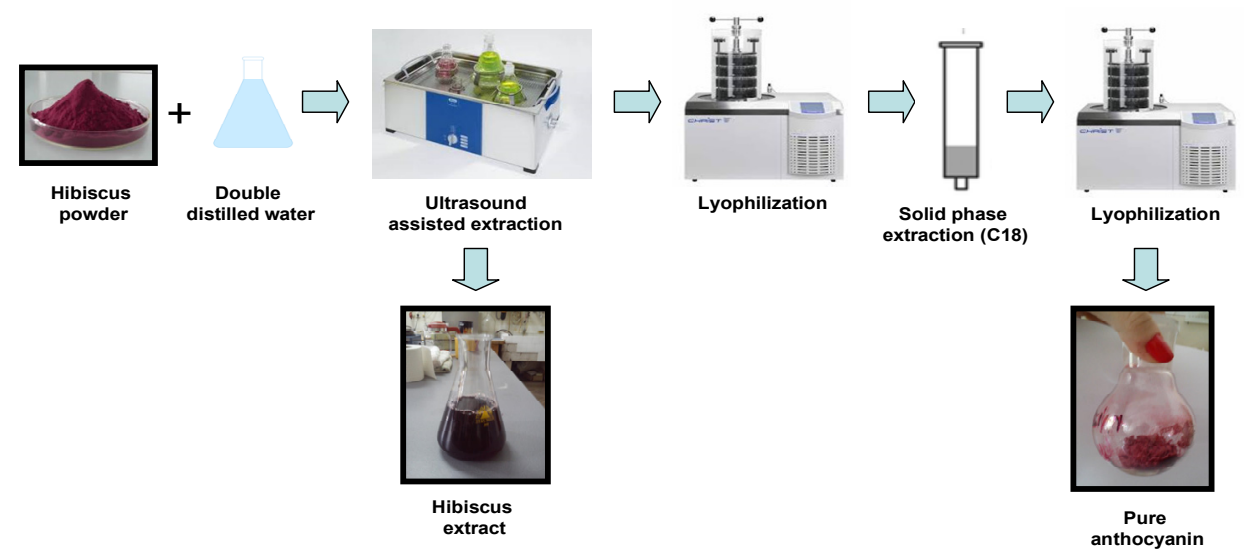

Figure 7. Schematic flowchart of anthocyanin extraction and separation from Hibiscus petals

The purified anthocyanin resulted from the above presented method will be denoted: $\mathrm{AEH}$.

Preparation of berry extract with AOX effect by ultrasound-assisted extraction method

Fruits used in this method were bio-products deriving from a natural environment (Satu-Mare and Sălaj counties, Romania); during the ripening process, they were not treated with herbicides, nor insecticides, and were not contaminated with any pollutant. The following berries were used in this study: blueberry (Vaccinium spp.), hawthorn (C. pinnatifida), blackthorn (Prunus spinosa L.), and blackcurrant (Ribes nigrum L.). An amount of $0.5 \mathrm{~g}$ smashed berries was mixed with $30 \mathrm{ml}$ of double distilled water in an Erlenmeyer flask. The flask was put in ultrasonic bath for 30 minutes at room temperature. The resulted solution was filtered by a vacuum filter. The filtrate containing anthocyanins and other compounds with possible AOX effect was kept in the refrigerator for 12 hours; after that the water excess was eliminated using a freeze-dryer. 
The resulted extract contains different types of anthocyanins characteristic for each type of berry, other types of polyphenols and carbohydrates, and it will be hereinafter referred to as berry AOX mixture. The exact amount of extract yield from different types of berries is summarized in Table 6 .

Table 6. Extracted amount of anthocyanins and polysaccharides (g) from different types of berries.

\begin{tabular}{|c|c|}
\hline Berries & $\begin{array}{c}\text { Extracted amount of anthocyanins and } \\
\text { polysaccharides }(\mathrm{g}) / \\
0.5 \mathrm{~g} \text { smashed berries }\end{array}$ \\
\hline Blueberry & $\mathbf{0 . 3 7 4}$ \\
\hline Blackcurrant & 0.357 \\
\hline Hawthorn & 0.335 \\
\hline Blackthorn & 0.257 \\
\hline
\end{tabular}

\section{Antioxidant effect measurements of the plant extract}

In order to compare the antioxidant activity of the extract of berries and hibiscus, and that of pure anthocyanin separated from hibiscus, the BR analytical method was used. The measurements were performed in a $20 \mathrm{ml}$ double-walled glass batch reactor connected to a FALC FA 90 thermostat (accuracy $\pm 0.1^{\circ} \mathrm{C}$ ) in order to maintain a constant $20^{\circ} \mathrm{C}$ temperature. A FALC 60 magnetic stirrer at a constant stirring rate provided continuous stirring. Oscillations were monitored with a double-junction saturated calomel electrode (SCE) as a reference electrode, and a Pt electrode. They were connected to a computer through a $\mathrm{PCl} 6036 \mathrm{E}$ data-acquisition interface. The oscillations were recorded through the LabView data-acquisition program and were processed by the means of Origin 9.0 program. Stock solutions of malonic acid (MA) (Aldrich, reagent grade, >99\%), manganese (II) sulfate monohydrate (Reactivul, reagent grade, $>99 \%$ ), and potassium iodate (Merck, reagent grade, $>99 \%$ ) were made without further purification of the reagents using double-distilled water. $\mathrm{H}_{2} \mathrm{SO}_{4}$ (Merck, 96\%) and $\mathrm{H}_{2} \mathrm{O}_{2}$ (Merck, 30\%) were of analytical grade. Hydrogen-peroxide was standardized by permanganometric analysis. The BR mixtures were obtained by mixing the appropriate amounts of stock solutions in a way that finally resulted in the initial composition $\left[\mathrm{KIO}_{3}\right]=$ $0.0268 \mathrm{M},\left[\mathrm{H}_{2} \mathrm{SO}_{4}\right]=0.0716 \mathrm{M},[\mathrm{MA}]=0.04 \mathrm{M},\left[\mathrm{MnSO}_{4}\right]=0.004 \mathrm{M},\left[\mathrm{H}_{2} \mathrm{O}_{2}\right]=$ $0.52 \mathrm{M}$. The order of mixing was: malonic acid, $\mathrm{MnSO}_{4}, \mathrm{H}_{2} \mathrm{SO}_{4}, \mathrm{KIO}_{3}$, and $\mathrm{H}_{2} \mathrm{O}_{2}$. Oscillations start after the addition of the $\mathrm{H}_{2} \mathrm{O}_{2}$. After the third oscillation $0.5 \mathrm{ml}$ diluted raw juice was added to the reactor by using a micropipette. 


\section{Preparation of silica-substituted hydroxyapatite (HAP-Si)}

HAP-Si was prepared by precipitation method, as described in previous studies [31, 32], under controlled conditions. The following materials were used: calcium nitrate tetrahydrate, diammonium phosphate, $25 \%$ ammonia solution (Alfa Aesar), and sodium-silicate. The resulted material (HAP-Si with $10 \mathrm{wt} \%$ Si) had $87 \mathrm{~m}^{2} / \mathrm{g}$ specific surface. The materials were not heat-treated, because based on previous studies [31], calcined materials were known to have lower retaining capacity.

\section{Adsorption measurements}

The adsorption process was monitored by a Jasco V650 spectrophotometer. In order to calculate the concentration of the extract, different dilutions of the extract were prepared (from a stock solution of $3 \mathrm{mg} / \mathrm{l}$ ) and the absorbance at specific wavelength $(\lambda=535 \mathrm{~nm})$ were plotted against the concentration of the extract. The adsorption measurements were carried out in thermostatic conditions (Falco FA90 thermostat), as follows: $0.05 \mathrm{~g} \mathrm{HAP-Si}$ material was added to a $10 \mathrm{ml}$ of berry solution with a concentration of $3 \mathrm{~g} / \mathrm{l}$ ( $0.03 \mathrm{~g}$ extract $+10 \mathrm{ml}$ water). The adsorption process was carried out in a brown beaker in order to avoid the degradation of anthocyanins caused by light. After 24 hours, the solution was centrifuged, separating the solid and liquid phase. The concentration was measured from the remaining solution using the UV-VIS spectrophotometer (V 530-Jasco) and then the adsorbed amount was calculated from the remaining anthocyanins in the solution. From this data, the adsorption capacity and adsorption rate were calculated, with the results presented in Table 4.

Adsorption capacity $\boldsymbol{q}_{t}(\mathrm{mg} / \mathrm{g})$ and adsorption efficiency $\boldsymbol{\eta}(\%)$ were calculated with equations (1) and (2), using the following formula:

$$
\begin{aligned}
& q_{t}=\frac{m_{\text {adsorbed }}}{m_{\text {adsorbent }}} \\
& \eta=\frac{c_{i}-c_{f}}{c_{i}} \cdot 100=\frac{c_{a d s}}{c_{i}} 100
\end{aligned}
$$

where: $m_{\text {adsorbed }}$ - adsorbed substance quantity $(g)$

$\mathrm{m}_{\text {adsorbent }}$ - adsorbent material quantity $(\mathrm{g})$

$\mathrm{C}_{\mathrm{i}}$-adsorbed substance initial concentration $(\mathrm{g} / \mathrm{L})$

$\mathrm{C}_{\mathrm{f}}-$ adsorbed substance final concentration $(\mathrm{g} / \mathrm{L})$

$\mathrm{C}_{\text {ads }}$ - adsorbed substance concentration $(\mathrm{g} / \mathrm{L})$ 


\section{REFERENCES}

1. J.A. Gomez del Rýo, P.J. Morando, D.S. Cicerone, Journal of Environmental Management, 2004, 71, 169.

2. H.Y. Xu, L. Yang, P. Wang, Y. Liu, M.S. Peng, Journal of Environmental Management, 2008, 86, 319.

3. F. Fernane, M.O. Mecherri, P. Sharrock, M. Hadioui, H. Lounici, M. Fedoroff, Materials Characterization, 2008, 59, 554.

4. H. Shi, H. Zhong, Y. Liu, J.Y. Deng, Acta Geologica Sinica -English Edition, 2007, 81, 517.

5. E.S. Bogya, R. Barabás, L. Bizo, V. R. Dejeu, Proceedings of the $11^{\text {th }}$ ECERS Conference, Krakow, 2009, 1109.

6. L.G. Ranilla, Y.-I. Kwon, E. Apostolidis, K. Shetty, Bioresource Technology, 2010, 101, 4676.

7. R. Nowak, M. Olech, N. Nowacka, "Polyphenols in Human Health and Disease", first ed., Academic Press, Elsevier, 2014, chapter 9.

8. A.J. Gomes Castro, L.S.E.P. Will Castro, M.S. Nascimento Santos, M.G.C. Faustino, T.S. Pinheiro, C.M.P. Guerra Dore, I.G. Baseia, E. Lisboa Leite, Biomedicine \& Preventive Nutrition, 2014, 4, 121.

9. L.-L. Huang, C. Pan, L. Wang, L. Ding, K. Guo, H.-Z.Wang, A.-M Xu, S. Gao, The Journal of Nutritional Biochemistry, 2015, 26, 841.

10. R. Fu, Y. Zhang, Y. Guo, T. Peng, F. Chen, Journal of Functional Foods, 2016, $22,73$.

11. V.M. Navarro Garcia, G. Rojas, L.G. Zepeda, M. Aviles, M. Fuentes, A. Herrera, E. Jiménez, Pharmaceutical Biology, 2006, 44, 297.

12. C. Zhang, Z. Gao, C. Hu, , J. Zhang, , X. Sun, C. Ronga, L. Jia, International Journal of Biological Macromolecules, 2017, 95, 778.

13. L. Bravo, Nutrition Reviews, 1998, 56, 317.

14. Y. Cai, Q. Luo, M. Sun, H. Corke, Life Sciences, 2004, 74, 2157.

15. Z.L. Ke, Y. Pan, X.D. Xu, C. Nie, Z.Q. Zhou, Journal of Food and Nutrition Research, 2015, 3, 341.

16. P. Rajendran, N. Nandakumar, T. Rengarajan, R. Palaniswami, E.N. Gnanadhas, U. Lakshminarasaiah, J. Gopas, I. Nishigaki, Clinica Chimica Acta, 2014, 436, 332.

17. J. Yu, L. Wang, R.L. Walzem, E.G. Miller, L.M. Pike, B.S. Patil, Journal of Agricultural and Food Chemistry, 2005, 53, 2009.

18. C. Grajeda-Iglesias, E. Salas, N. Barouh, B. Baréa, A. Panya, M.C. FigueroaEspinoza, Food Chemistry, 2016, 194, 749.

19. K.M. Schaich, X. Tian, J. Xie, Journal of Functional Foods, 2015, 14, 111.

20. B. Du, B. Xu, Bioactive Carbohydrates and Dietary Fibre, 2014, 3(1), 11.

21. N. Muntean, G. Szabó, M. Wittmann, T. Lawson, J. Fülöp, Z. Noszticzius, L. Onel, The Journal of Physical Chemistry A, 2009, 113, 9102.

22. R. Cervellati, K. Höner, S.D. Furrow, D. Stanley, C. Neddens, S. Costa, Helvetica Chimica Acta, 2001, 84, 3533. 
23. R. Cervellati, E. Greco, Helvetica Chimica Acta, 2016, 99, 41.

24. N. Muntean, G. Szabó, Studia UBB Chemia, 2013, LVII, 2, 175.

25. N. Muntean, G. Szabó, Studia UBB Chemia, 2015, LX, 3, 273.

26. M. Li, G. Hu, Y. Chen, Food Chemistry, 2016, 197, 987.

27. D. Skroza, I. Generalić Mekinić, S. Svilović, V. Šimat, V. Katalinić, Journal of Food Composition and Analysis, 2015, 38, 13.

28. T. Kokubo, H. Takadama, Biomaterials, 2006, 27, 2907.

29. T. Kokubo, S. Ito, M. Shigematsu, S. Sanka, T. Yamamuro, Journal of Materials Science, 1987, 22, 4067

30. J. Oszmianski, C.Y. Lee, American Journal of Enology and Viticulture, 1990, 41, 204.

31. E.S. Bogya, R. Barabás, V.R. Dejeu, Al. Csavdari, I. Bâldea, Chemical Papers, 2009, 63, 568.

32. Al.-C. Dancu, R. Barabás, E.S. Bogya, Central European Journal of Chemistry, 2011, 9, 660 . 